\title{
CASE REPORT: ACUTE FATTY LIVER OF PREGNANCY
}

\author{
O Austin-Chukwu, SpR, Obstetrics and Gynaecology \\ Furness General Hospital
}

A 30-year-old primigravida presented at 33 weeks with abdominal pain and a blood pressure of $122 / 90 \mathrm{mmHg}$. She had booked at $8 \frac{1}{2} 2$ weeks' gestation and the pregnancy had remained uncomplicated until now. Although she had no urinary symptoms, urinalysis showed moderate leucocytes. She was admitted for observation, a midstream specimen of urine was sent for culture and she was started on oral antibiotics for a suspected urinary tract infection (UTI).

She felt better the following day and her blood pressure remained stable at $120 / 80$. She was discharged. She presented ten days later with reduced fetal movements and vague symptoms of abdominal pain, backache and vomiting. She was mildly pyrexial and was thus started on intravenous Augmentin for a suspected UTI.

At this stage, she was noted on routine blood testing to have elevated liver enzymes (ALP 970u/l; ALT 140u/l; Bilirubin 38u/l; reference values: 70-300u/l, $<38 \mathrm{u} / \mathrm{l}$ and $<20 \mathrm{umol} / \mathrm{l}$ respectively). The haematological indices were within the normal range. As the urine culture did not grow any organisms, antibiotics were stopped. She continued to feel unwell and complained of reduced fetal movements despite normal cardiotocograph tracings (CTG). An ultrasound scan was performed and showed the appropriate foetal size for gestation, adequate liquor volume, and a normal Doppler. Repeat biochemical tests showed that there was a further rise in the liver enzymes. A viral hepatitis screen was carried out.

She was assessed by the medical team who made a provisional diagnosis of acute fatty liver of pregnancy (AFLP). She was started on $40 \mathrm{mg}$ of prednisolone daily with the intention to deliver the baby within 24 hours. The plan was to induce labour the following day with Prostin, but the patient had spontaneous rupture of the membranes at 0610 hours.

On examination she was $3-4 \mathrm{cms}$ dilated and it was decided to augment the labour with syntocinon in order to expedite delivery. The liver enzymes continued to rise and by 1200 hours were: ALP 957; ALT 1049. An emergency Caesarean section was carried out. She was delivered of a live female infant weighing $2.030 \mathrm{~kg}$ and with Apgar scores of 9 at one minute and five minutes.

The liver enzymes continued to rise and on her first postoperative day she was noted to have grade one encephalopathy and an enlarged but non-tender liver. An ultrasound scan of the liver was requested and this showed mild hepatomegaly but no focal parenchymal lesions. Intravenous infusion with $5 \%$ glucose was maintained for the first three days.

By the second postoperative day, the liver enzymes had achieved a plateau (ALP 726; ALT 1352) and then began to fall. Coagulation screen had remained normal until the first post-operative day when the APTT ratio was 9.1 (reference range: 0.9-1.15) and fibrinogen levels were decreased at $1.00 \mathrm{~g} / \mathrm{l}$ (reference range: $2.0-4.0$ ). The viral hepatitis screen was negative for hepatitis $\mathrm{A}, \mathrm{B}$ and $\mathrm{C}$.

She was treated with oral Amoxycillin for an E coli UTI on the fifth postoperative day and transfused with two units of packed cells on the seventh postoperative day because she had a haemoglobin of $8.4 \mathrm{~g} / \mathrm{l}$. She was discharged on the eleventh postoperative day (ALP 522; ALT 253). At her six-week postnatal check, Mum and baby were doing well.

\section{DISCUSSION}

AFLP is a rare and potentially fatal condition, usually occurring in the third trimester of pregnancy. Its exact incidence is unknown because many cases go unreported, but is in the region of $1: 13,328$ pregnancies $^{(1)}$. Although the definitive way of making the diagnosis is by liver biopsy, this carries considerable risk of bleeding because of the abnormal coagulation which is a frequent component of the condition. In most cases, a high level of suspicion based on clinical presentation and supported by biochemical investigations is enough to make a diagnosis. Ultrasound and CT scan may be of help but are associated with high false negative rates. ${ }^{(2)}$

In our patient, abnormal liver enzymes were found on routine blood tests in a woman with mildly raised blood pressure and non-specific abdominal symptoms. The diagnosis of AFLP was made on clinical grounds and laboratory investigations alone and this is acceptable practice $^{(3)}$. It is not usual to find such high level liver transaminases in AFLP as we found in our patient. This is commoner in acute viral hepatitis ${ }^{(4)}$ but a negative screen ruled out this diagnosis. Similarly, HELLP syndrome (haemolysis, elevated liver enzymes, low platelets) was ruled out because of normal platelets, normal blood pressure and no other signs to suggest pre-eclampsia.

After the diagnosis of AFLP is made, maternal stabilisation and expedition of delivery is advocated ${ }^{(1)}$. Our patient illustrates that such management can avoid the development of hepatic failure and extrahepatic disease. A fairly quick fall of the liver enzymes following delivery was seen in this case and is to be expected.

Abnormal coagulation in the form of prolonged prothrombin time (PT) and decreased fibrinogen is a common finding in AFLP but this generally occurs in severe cases. In our patient the only evidence of disturbed coagulation was a transiently increased activated partial thromboplastin time (APTT) and reduced fibrinogen. 
In retrospect a liver biopsy carried out postpartum would have been of benefit in this patient with an unusual presentation to confirm the diagnosis. This is important for future pregnancies as recurrence has been reported. It appears that like most other conditions, there are varying levels of severity in AFLP and it is invariably in the mild atypical cases that the diagnosis is missed. This could easily have happened in our patient who did not present with the usual myriad of symptoms and signs seen in AFLP. A high index of suspicion and prompt instigation of laboratory investigations will certainly help in reducing the number of cases that go undiagnosed with serious consequences.

\section{REFERENCES}

1 Watson WJ, Seeds JW Acute fatty liver of pregnancy Obstet Gynecol Surv 1990;45:585-593

2 Van de L, Podrasky A Computed tomography and ultrasonic findings in women with acute fatty liver of pregnancy J Reprod Med 1996;35:815-817

3 Castro MA, Ouzounian JG, Colletti PM et al Radiologic studies in acute fatty liver of pregnancy. A review of the literature and 19 new cases. J Reprod Med 1996;41:839-843 4 Yannick Bacq Acute fatty liver of pregnancy Seminars in Perinatology 1998;22(2):134-140 